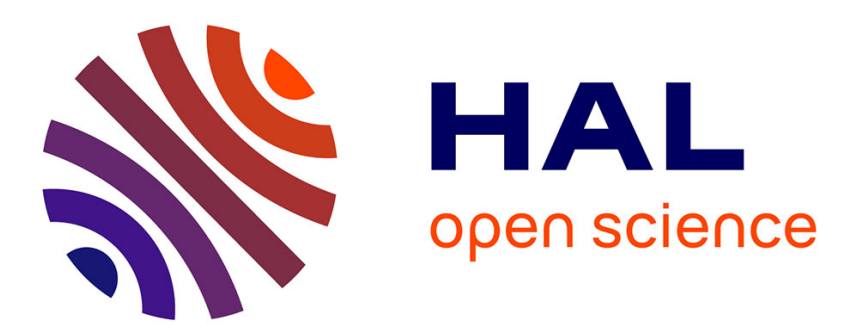

\title{
Traitement par voie endovasculaire d'un faux anévrisme tuberculeux de l'isthme aortique
}

O Steichen, O Pellerin, M Frank, J Emmerich, M. Sapoval, J.-N Fiessinger, A Bura-Rivière

\section{- To cite this version:}

O Steichen, O Pellerin, M Frank, J Emmerich, M. Sapoval, et al.. Traitement par voie endovasculaire d'un faux anévrisme tuberculeux de l'isthme aortique. La Revue de Médecine Interne, 2007, 28 (3), pp.196-198. 10.1016/j.revmed.2006.11.017 . hal-01171802

\section{HAL Id: hal-01171802 https://hal.sorbonne-universite.fr/hal-01171802}

Submitted on 6 Jul 2015

HAL is a multi-disciplinary open access archive for the deposit and dissemination of scientific research documents, whether they are published or not. The documents may come from teaching and research institutions in France or abroad, or from public or private research centers.
L'archive ouverte pluridisciplinaire HAL, est destinée au dépôt et à la diffusion de documents scientifiques de niveau recherche, publiés ou non, émanant des établissements d'enseignement et de recherche français ou étrangers, des laboratoires publics ou privés. 


\section{Traitement par voie endovasculaire d'un faux anévrysme tuberculeux de l'isthme aortique}

O. Steichen * $(1,4)$; O. Pellerin $(2,4)$; M. Frank $(3,4)$; J. Emmerich $(3,4)$; M. Sapoval $(2,4)$; J.-N. Fiessinger $(3,4)$; A. Bura-Rivière $(3,4)$.

(1) INSERM, U729 «Ingénierie des connaissances en santé », Laboratoire de Santé Publique et d'Informatique Médicale, Institut de Recherche des Cordeliers, 15 rue de l'Ecole de Médecine, F-75006 Paris, France ;

(2) Assistance Publique - Hôpitaux de Paris, Hôpital Européen Georges Pompidou, Service de Radiologie Cardiovasculaire, 20 rue Leblanc, F-75015 Paris, France ;

(3) Assistance Publique - Hôpitaux de Paris, Hôpital Européen Georges Pompidou, Service de Médecine Vasculaire et Hypertension Artérielle, 20 rue Leblanc, F-75015 Paris, France ;

(4) Université Paris Descartes, Faculté de Médecine, Hôpital Européen Georges Pompidou, 20 rue Leblanc, F-75015 Paris, France.

* auteur correspondant :

Laboratoire Spim - Inserm U729, Institut de Recherche des Cordeliers, 15 rue de l'Ecole de Médecine, 75006 Paris,

tel. 01423469 83, fax 01531092 01, mél. ost@ club-internet.fr 


\section{Résumé}

\section{Introduction}

Les anévrysmes aortiques représentent une localisation classique mais rare de la tuberculose. Leur complication la plus grave est la rupture, imprévisible et létale.

\section{Exégèse}

Des douleurs thoraciques et des crachats hémoptoïques ont révélé un faux anévrysme de l'isthme aortique chez un homme de 48 ans. Dans le cadre de l'urgence, une prothèse aortique a été mise en place par voie endovasculaire. Le diagnostic de tuberculose a été porté après 6 semaines par la culture des tubages gastriques et un traitement médical a alors été mis en route.

Les anévrysmes aortiques tuberculeux sont le plus souvent de faux anévrysmes, secondaires à l'érosion de la paroi aortique par un foyer tuberculeux adjacent. Ils sont révélés par des douleurs, des manifestations hémorragiques ou un syndrome de masse para-aortique.

\section{Conclusion}

Les faux anévrysmes aortiques tuberculeux justifient une intervention rapide pour éviter leur rupture. Le traitement de référence est chirurgical mais la mise en place d'une prothèse par voie endovasculaire est une alternative thérapeutique, notamment dans les situations à fort risque opératoire.

\section{Mots-clés}

Tuberculose cardiovasculaire, anévrysme de l'aorte thoracique, faux anévrysme, stent, évaluation résultats traitement 


\section{Title}

Endovascular repair of a tuberculous aortic false aneurysm

\section{Abstract \\ Introduction}

Aortic aneurysms are a well known but rare complication of tuberculosis. Their major complication is aneurysmal rupture, unforeseeable and lethal.

\section{Exegesis}

Chest pain and hemoptoic expectoration revealed a false aneurysm of the aortic isthmus in a 48-year-old man. Endovascular repair with a stent graft was urgently undertaken. Tuberculosis was diagnosed 6 weeks thereafter by the growth of gastric juice cultures and medically treated.

Most tuberculous aortic aneurysms are false aneurysms, caused by an adjacent tuberculous focus eroding the aortic wall. They present with pain, bleeding or as para-aortic masses.

\section{Conclusion}

Tuberculous false aneurysms of the aorta necessitate an early intervention before they rupture. Surgical treatment remains the preferred option but endovascular repair with a stent graft is a therapeutic alternative, to be considered in high-risk surgical patients.

\section{Keywords}

Cardiovascular tuberculosis, thoracic aortic aneurysm, false aneurysm, stents, treatment outcome 


\section{Introduction}

Les anévrysmes aortiques constituent une localisation classique mais rare de la tuberculose, dont le pronostic est dominé par le risque de rupture, conduisant invariablement au décès du patient [1]. La prise en charge de référence associe une réparation chirurgicale et un traitement antituberculeux. Nous présentons un cas traité avec succès par voie endovasculaire, avant l'obtention du diagnostic étiologique.

\section{Observation}

Un homme de 48 ans d'origine vietnamienne a consulté pour un crachat hémoptoïque compliquant une toux sèche associée à des douleurs thoraciques et à une dysphonie depuis 10 jours. Il n'avait pas d'antécédent médical ou traumatique. Il avait perdu $15 \mathrm{~kg}$ depuis son arrivée en France 17 ans auparavant, probablement en raison d'un changement de régime alimentaire. La chronologie des variations pondérales n'était pas connue. Le patient était apyrétique et l'examen clinique était strictement normal. Les examens biologiques usuels n'ont montré qu'un syndrome inflammatoire modéré avec une protéine C-réactive (PCR) à $20 \mathrm{mg} / \mathrm{l}$. Le scanner avec injection a révélé un volumineux faux anévrysme de l'isthme aortique comblant la fenêtre aortopulmonaire, de $50 \mathrm{~mm}$ de plus grand diamètre (Figure 1.A). L'aorte était saine, en dehors de l'ulcération à l'origine du faux anévrysme. Il existait une discrète condensation pulmonaire en regard de la lésion, probablement liée à une suffusion hémorragique, mais pas d'autre anomalie pleuroparenchymateuse ou médiastinale. En raison du risque de rupture défini par la taille de l'anévrysme et par l'hémoptysie, une endoprothèse aortique a été mise en place en urgence par voie fémorale, couvrant l'ulcération pariétale afin d'exclure le faux anévrysme (Figure 1.B).

La recherche d'une cause d'aortite est restée infructueuse (hémocultures; sérologies du virus de l'immunodéficience humaine, de la syphilis, des mycoplasmes, des chlamydias, de la légionelle, des bartonelles et des rickettsies; facteur rhumatoïde, anticorps antinucléaires, anti-antigènes nucléaires solubles, antiphospholipides et anticytoplasme des polynucléaires). L'intradermoréaction (IDR) à 5 unités de tuberculine était positive, avec un diamètre de $15 \mathrm{~mm}$ à la $72^{\text {ème }}$ heure. Le statut vaccinal et l'existence d'éventuelles IDR antérieures étaient inconnus. Un examen oto-rhino-laryngologique a permis de rattacher la dysphonie à une paralysie récurrentielle gauche, liée à une irritation par le faux anévrysme. La fibroscopie bronchique, réalisée après trois tubages gastriques négatifs à l'examen direct, était normale.

Six jours après la mise en place de l'endoprothèse, le patient était cliniquement stable et le scanner de contrôle confirmait l'exclusion du pseudo-anévrysme. L'indication d'une intervention chirurgicale n'a donc pas été retenue, y compris à visée diagnostique. Le retour au domicile a été autorisé avec un traitement antiagrégant par aspirine $(160 \mathrm{mg} / \mathrm{j})$. Des rendez-vous de consultation mensuels ont été planifiés avec une surveillance biologique systématique. Un scanner thoracique de contrôle a été prévu à deux mois de l'intervention.

Le diagnostic de tuberculose a finalement été porté après 6 semaines par le résultat de la culture des tubages gastriques (Mycobacterium tuberculosis). À ce moment, le patient était toujours cliniquement stable et la PCR s'était normalisée spontanément. Le scanner de contrôle demeurait sans autre particularité que le faux anévrysme, en voie de régression à $37 \mathrm{~mm}$ de plus grand diamètre. Un traitement antituberculeux a été instauré, associant isoniazide, rifampicine, éthambutol et pyrazinamide. Un mois plus tard, l'antibiogramme a démontré la sensibilité de la souche à tous les antituberculeux majeurs et l'éthambutol a été arrêté. Le pyrazinamide a été interrompu après 2 mois, alors que l'isoniazide et la rifampicine ont été administrés pour une durée totale de 6 mois. À l'issue du traitement, le 
patient avait repris $6 \mathrm{~kg}$, la dysphonie avait disparu et le faux anévrysme avait complètement régressé (Figure 1.C).

\section{Discussion}

Une recherche dans Medline avec les mots-clés du Medical Subject Headings $(\mathrm{MeSH})$ 《tuberculosis » et «aneurysm » permet de trouver une revue de la littérature publiée par Long et al. en 1999, qui collige 41 cas d'anévrysmes aortiques tuberculeux rapportés entre 1945 et 1999 [1], et une vingtaine de cas supplémentaires publiés entre 1999 et 2006. Ces nouveaux cas ne remettent pas en cause les conclusions physiopathologiques, diagnostiques et thérapeutiques de la revue, résumées dans les paragraphes suivants.

Quatre-vingt-dix pour cent des anévrysmes aortiques tuberculeux n'ont pas de paroi propre et sont donc des faux anévrysmes développés à partir d'une perforation de la paroi artérielle. Ils sont limités par les structures anatomiques de voisinage et par du matériel thrombotique plus ou moins organisé. Dans $75 \%$ des cas, l'érosion pariétale est secondaire à un foyer tuberculeux extravasculaire au contact de l'aorte. Seuls les $25 \%$ restant sont des anévrysmes mycotiques à proprement parler. La greffe bacillaire sur la paroi artérielle se produit alors soit directement par la lumière aortique, soit par l'intermédiaire des vasa vasorum.

Les anévrysmes tuberculeux se répartissent également entre l'aorte thoracique et l'aorte abdominale. Trois modes de révélation sont possibles: syndrome douloureux (thoracique, abdominal ou rachidien), syndrome hémorragique (choc ou extériorisation) ou syndrome de masse para-aortique (clinique ou radiologique). Dans presque la moitié des cas, le diagnostic est porté dans le cadre d'une tuberculose disséminée.

Aucun patient ayant survécu en l'absence d'une association thérapeutique médicochirurgicale n'a été identifié par la revue de Long et al. [1]. La rupture du faux anévrysme est imprévisible, le traitement chirurgical est donc indiqué aussi rapidement que possible. La chirurgie est urgente en cas de manifestations hémorragiques et pour les volumineux faux anévrysmes (supérieurs à $3 \mathrm{~cm}$ de diamètre). L'intervention consiste en un débridement des tissus infectés suivi d'une reconstruction prothétique in situ ou plus rarement d'un pontage extra-anatomique.

Un traitement antituberculeux est systématiquement associé, mais sa durée optimale n'est pas définie. Par analogie avec les autres localisations de la maladie tuberculeuse, cette durée est au minimum de 6 mois. La supériorité d'un traitement plus long n'a jamais pu être démontrée pour aucune localisation, même s'il est habituel de traiter plus longtemps certaines formes graves, en particulier cérébro-méningées [2]. Dans le cas rapporté ici, il n'a pas été jugé nécessaire de prolonger le traitement malgré la mise en place de matériel étranger, compte tenu de la faible charge bacillaire initiale et de l'évolution rapidement favorable.

Bien que le traitement de référence soit chirurgical, l'observation présentée illustre la possibilité d'un traitement par voie endovasculaire, déjà décrite pour deux anévrysmes tuberculeux de l'aorte abdominale [3]. Les résultats à moyen terme sont encourageants avec 18 et 24 mois de recul pour les cas abdominaux, et 12 mois pour notre cas thoracique. Un cas a également été rapporté au niveau de l'artère fémorale superficielle mais le patient a succombé à un infarctus du myocarde un mois après l'intervention [4].

L'expérience est encore insuffisante pour dire si les résultats à long terme sont équivalents à ceux de la chirurgie et le traitement endovasculaire doit être réservé à des cas particuliers. En effet, l'habitude persiste de ne pas poser de matériel étranger en zone infectée sans débridement préalable. L'observation présentée se singularise par l'absence de foyer infectieux patent au moment du diagnostic. En l'absence d'orientation étiologique, le 
traitement par voie endovasculaire a été préféré pour éviter au patient la lourdeur d'une intervention avec thoracotomie.

Le dogme chirurgical commence à être remis en question pour les anévrysmes aortiques infectieux non tuberculeux (essentiellement streptocoques, staphylocoques et salmonelles). Le nombre d'interventions endovasculaires réalisées dans ce cadre est plus important, avec un plus grand recul. Des données récentes suggèrent que cette modalité thérapeutique pourrait obtenir des résultats comparables à ceux de la chirurgie [5]. À l'heure actuelle, le traitement par voie endovasculaire des anévrysmes aortiques infectieux est à considérer lorsque l'accès à la chirurgie cardiovasculaire est difficile et pour les patients à risque opératoire élevé.

\section{Conclusion}

Les anévrysmes aortiques tuberculeux, comme tous les faux anévrysmes aortiques, justifient une intervention rapide avant leur rupture. Le traitement chirurgical fait toujours référence mais les nouvelles techniques de radiologie interventionnelle, grevées d'une moindre mortalité périopératoire, rendent possible une prise en charge moins invasive. L'observation présentée illustre la faisabilité d'un traitement par voie endovasculaire. Toutefois, il n'existe aucune étude comparant le traitement endovasculaire au traitement chirurgical conventionnel en terme de morbi-mortalité à long terme dans le cadre des anévrysmes aortiques tuberculeux. En attendant de définir plus clairement les indications interventionnelles, le traitement par voie endovasculaire peut être considéré en cas de contre-indication chirurgicale ou de risque opératoire élevé. 


\section{Références}

1. Long R, Guzman R, Greenberg H, Safneck J, Hershfield E. Tuberculous mycotic aneurysm of the aorta: review of published medical and surgical experience. Chest 1999; 115:522-31.

2. Conseil Supérieur d'Hygiène Publique. Prévention et prise en charge de la tuberculose en France. Synthèse et recommandations du groupe de travail du Conseil Supérieur d'Hygiène Publique en France. Rev Mal Respir 2003; 20:S3-S106.

3. Liu WC, Kwak BK, Kim KN, Kim SY, Woo JJ, Chung DJ et al. Tuberculous aneurysm of the abdominal aorta: endovascular repair using stent grafts in two cases. Korean J Radiol 2000; 1:215-8.

4. Lagattolla N, Baghai M, Biswas S, Redington A, Downie A, Miburn H et al. Tuberculous false aneurysm of the femoral artery managed by endoluminal stent graft insertion. Eur J Vasc Endovasc Surg 2000; 19:440-2.

5. Jones KG, Bell RE, Sabharwal T, Aukett M, Reidy JF, Taylor PR. Treatment of mycotic aortic aneurysms with endoluminal grafts. Eur J Vasc Endovasc Surg 2005; 29:139-44. 
Figure 1. Angioscanner de l'aorte thoracique en coupes transversales à la hauteur du point de fuite (isthme aortique, images de gauche) et du diamètre maximal de l'anévrysme (bifurcation trachéale, images de droite).

A. Au moment du diagnostic : fuite de produit de contraste dans le faux anévrysme (flèche).

B. Après la mise en place de l'endoprothèse : colmatage de la fuite et exclusion du faux anévrysme.

C. Après 6 mois de traitement antituberculeux : résolution du faux anévrysme.

A
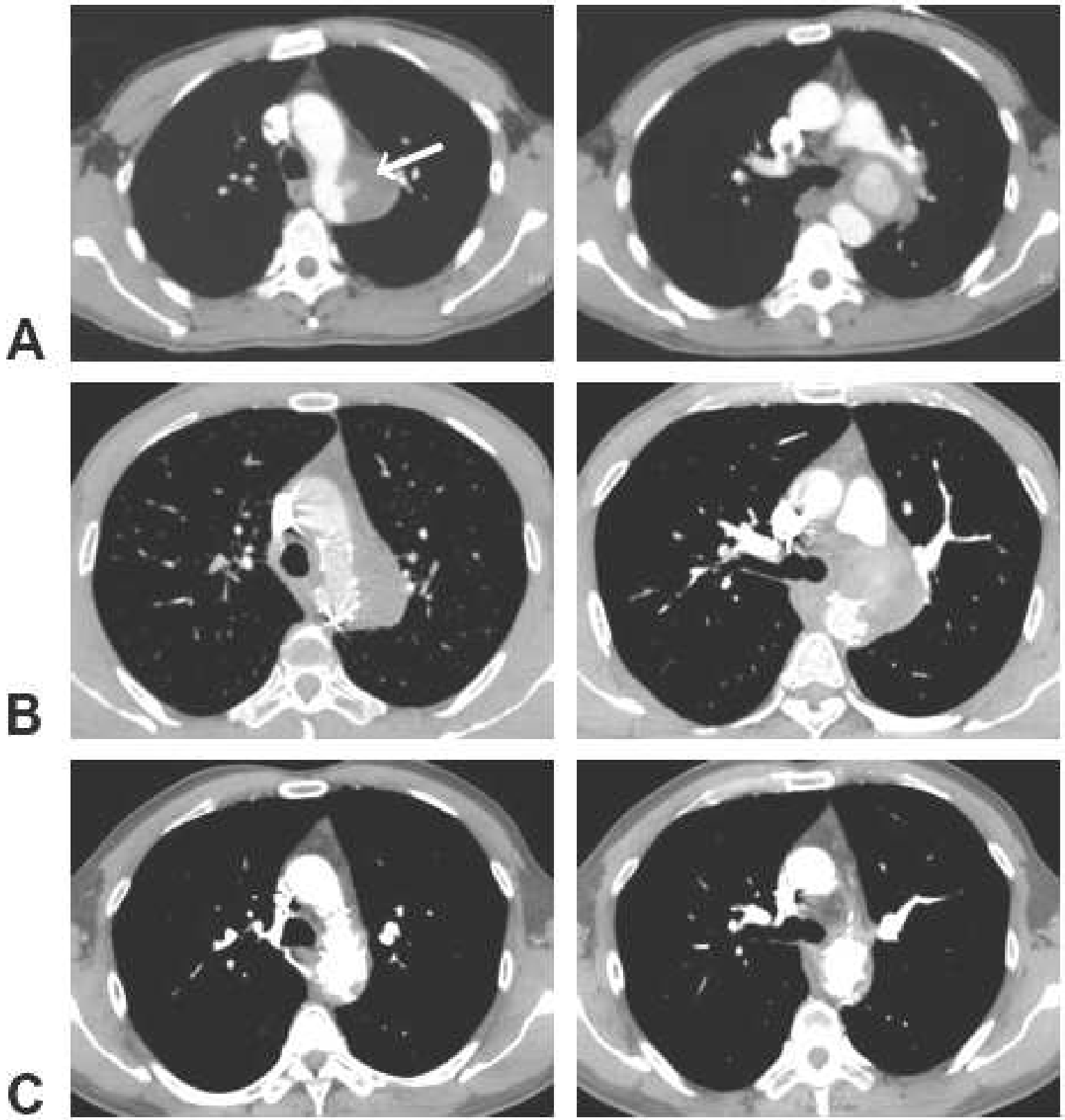